\title{
PARTICIPACIÓN LEGISLATIVA EN LA CONSTRUCCIÓN DE DERECHOS DE PROPIEDAD INTELECTUAL ${ }^{1}$
}

\author{
Sindy Mora Solano*
}

RESUMEN

En este artículo se analiza la participación legislativa en la construcción de derechos de propiedad intelectual, específicamente los referidos a la propiedad de semillas mejoradas de plantas y que son requisitos del Tratado de Libre Comercio entre Centroamérica y Estados Unidos (TLC). Se analiza cómo al interior de la Asamblea Legislativa, la diferenciación social se convierte en desigualdad en la búsqueda de espacios para la discusión legislativa.

PALABRAS CLAVE: COSTA RICA * PROPIEDAD INTELECTUAL * ASAMBLEA LEGISLATIVA * PARTICIPACIÓN LEGISLATIVA * ORGANIZACIONES SOCIALES * COMUNIDADES EPISTÉMICAS

\section{ABSTRACT}

This article analyses the legislative participation in the construction of intellectual property rights, specifically those referring to the property of improved plant varieties, which are a requisite for the Central America Free Trade Agreement (CAFTA). Research findings show that social differentiation is expressed as inequity in the search of spaces for legislative discussion.

KEY WORDS: COSTA RICA * INTELLECTUAL PROPERTY * ASAMBLEA LEGISLATIVA * LEGISLATIVE PARTICIPATION $*$ SOCIAL ORGANIZATIONS $*$ EPISTEMIC COMMUNITY

\section{INTRODUCCIÓN}

A partir de 1995, con el surgimiento de la Organización Mundial del Comercio (OMC) entró en vigencia el Acuerdo sobre los Aspectos de los Derechos de Propiedad Intelectual relacionados con el Comercio (ADPIC). Este acuerdo, cuya ratificación fue un requisito de ingreso a la OMC, se convirtió en el principal instrumento de regulación de la propiedad intelectual al nivel internacional.

Para cumplir con las disposiciones del ADPIC, la administración Rodríguez Echeverría (1998-2002) puso en la corriente legislativa una

\footnotetext{
* Instituto de Investigaciones Sociales, Universidad de Costa Rica. smora@iis.ucr.ac.cr

1 El presente artículo forma parte de un trabajo de investigación mayor titulado "Relaciones de poder en las disputas legislativas por la construcción de derechos de propiedad intelectual sobre variedades mejoradas de plantas". Esta investigación fue financiada con una beca para investigadores del Consejo Latinoamericano de las Ciencias Sociales (CLACSO).
} 
serie de proyectos de ley. En 1999 se instaló en la Asamblea Legislativa una comisión especial cuya función fue reformar la legislación existente en el ámbito de la propiedad intelectual. Presidida por Belisario Solano, diputado del Pusc, dicha comisión se denominó "Comisión Especial Nombrada para que estudie, analice y dictamine la legislación que sobre propiedad intelectual requiere nuestro país para enfrentar los desafíos y compromisos internacionales asumidos a la fecha" ${ }^{2}$ (Asamblea Legislativa, Expediente 13756: 64).

Para diciembre de 1999, esta comisión había aprobado un total de 7 propuestas legislativas. Debido a la rápida tramitación y la poca discusión con la que fueron aprobados estos proyectos he denominado a este proceso el "Combo de Propiedad Intelectual", haciendo alusión a la aprobación de los proyectos de "Ley para el Mejoramiento de los Servicios Públicos de Electricidad y Telecomunicaciones y de la Participación del Estado" que serían aprobados en primer debate solo tres meses después, y que popularmente fueron conocidos como "Combo ICE" .

No obstante, la Comisión de Propiedad Intelectual no pudo aprobar dos iniciativas. Estas fueron el proyecto de ley de Protección a las Obtenciones Vegetales y el proyecto del Convenio Internacional de Protección a las Obtenciones Vegetales, conocido como Convenio UPOV. Ambos proyectos se basan en una ley tipo establecida por la Unión Internacional para la Protección a las Obtenciones Vegetales (UPOV), entidad internacional

2 En adelante denominaré a esta instancia legislativa "Comisión de Propiedad Intelectual".

Forman parte del "Combo de la Propiedad Intelectual" la Ley de Protección a los Sistemas de Trazado de Circuitos Integrados; el Tratado de la OMPI sobre Interpretación y Ejecución de Fonogramas y sobre Derechos de Autor; la Ley de Marcas y Signos Distintivos; las reformas a la Ley de Derechos de Autor y Derechos Conexos; las reformas a la Ley de Patentes de Invención, Dibujos y Modelos Industriales y Modelos de Utilidad; la Ley de Información No Divulgada y la Ley de Procedimientos de Observancia de los Derechos de Propiedad Intelectual. Siete de estos ocho proyectos se aprobaron entre el 13 y el 22 de diciembre de 1999 (Expedientes legislativos, nro. 13156, nro. 13157, nro. 13641, nro. 13642, nro. 13643, nro. 13644 y nro. 13711). encargada de vigilar los derechos de propiedad intelectual sobre variedades mejoradas de plantas, siendo su finalidad el regular los derechos de propiedad intelectual sobre la mejora de semillas, actividad conocida como fitomejoramiento. Mientras que el primero supone la regulación nacional de este derecho, el segundo refiere al marco internacional para su cumplimiento, siendo su aprobación parte de los requisitos de la ratificación del Tratado de Libre Comercio entre Centroamérica y Estados Unidos (TLC).

Ante la puesta en corriente legislativa de ambos proyectos, el 13 de noviembre del 2003 diversas organizaciones sociales agrupadas en la Red de Coordinación en Biodiversidad presentaron el proyecto de ley de Protección de los Derechos de los Fitomejoradores. Sin embargo a finales del 2005, ninguna de las propuestas había sido aprobada ${ }^{4}$. Considerando lo anterior, la presente reflexión tiene como objetivo analizar la participación legislativa en la construcción de derechos de propiedad intelectual sobre semillas mejoradas, por lo que constituye un análisis de la participación de un proceso aún inconcluso. Sin embargo, los datos recabados hasta el momento permiten corroborar el supuesto de que la Asamblea Legislativa se construye y se estructura como espacio de participación diversa y desigual.

\section{¿QUÉ ES LO QUE ESTÁ EN JUEGO \\ EN LA CONSTRUCCIÓN DE DERECHOS DE PROPIEDAD INTELECTUAL?}

La construcción de derechos de propiedad intelectual de variedades mejoradas de plantas no es solo una disputa de carácter jurídico. Esto significa que en las discusiones legislativas no se discute únicamente qué tipo de derecho se busca crear, sino quiénes y cómo participan en la construcción de este derecho. Por ello, los distintos participantes deben emprender una lucha por acceder a los recursos disponibles en la Asamblea Legislativa e incidir en la toma de decisiones.

4 1999-2005 es el periodo abarcado por la investigación. Si bien desconozco los detalles de la tramitación parlamentaria de estos proyectos para el 2006, los mismos se encuentran sin aprobar todavía. 
Considerando lo anterior, la disputa legislativa analizada se compone en primer lugar por el objeto de la disputa, que refiere a aquello sobre lo que buscan regular los proyectos. El objeto de la disputa refiere a la construcción del derecho de propiedad intelectual sobre variedades mejoradas de plantas, derecho conocido como derecho del fitomejorador u obtentor. Un segundo elemento de la disputa refiere al mecanismo de regulación que debe ser aprobado, ya sean los proyectos de ley gubernamentales basados en la ley UPOV o la propuesta de la Red de Coordinación en Biodiversidad, lo que responde a la pregunta de ¿cómo se debe legislar? Pero para que se reconozca un determinado tipo de protección intelectual es necesario dar cuenta de un tercer nivel de la disputa, que es la participación en las discusiones legislativas de los proyectos de ley y el proceso de incidencia en la toma de decisiones. Al preguntar sobre quiénes y cómo inciden en la discusión y aprobación de los proyectos de ley, se busca atender a las disputas por acceder al ámbito legislativo con el fin de incidir en la toma de decisiones legislativas.

Si bien es necesario distinguir los tres niveles de la disputa, estos no se pueden comprender de manera separada, ya que para buscar el reconocimiento de derechos de propiedad intelectual sobre las semillas mejoradas, los sujetos con diversidad de estrategias y recursos deben incidir en el proceso de toma de decisiones legislativas, apoyando u oponiéndose a la aprobación de los proyectos de ley.

\section{¿CÓMO PARTICIPAR EN LA ASAMBLEA LEGISLATIVA?}

La participación en la Asamblea Legislativa puede ser de dos tipos, ya sea la participación por la vía escrita o la participación en audiencia. Estas formas de participar son a la vez los principales recursos que los sujetos deben disputarse para ingresar al escenario legislativo y expresar allí sus posiciones.

Estas formas de participación se encuentran definidas por el Reglamento de la Asamblea Legislativa. Los diputados pueden extender consultas escritas o convocar a audiencia a los representantes de instituciones estatales, quienes están en la obligación de responder al llamado legislativo. Sin embargo, cuando no se trata de representantes gubernamentales, los diputados deben presentar mociones que son discutidas en las comisiones legislativas, con el fin de determinar si es pertinente extender la invitación o no (Asamblea Legislativa, 2001: 93-97). En ocasiones, este procedimiento se convierte en un mecanismo de filtro de los sujetos a los que resulta adecuado consultar. Por ello la utilización de estos recursos depende no solo de la voluntad de los legisladores, sino también de la tenacidad $y$ en algunos casos insistencia de los sujetos para ser escuchados por los diputados.

Sobre la participación por la vía escrita solo habría dos posibilidades que son: ser consultados por la vía escrita o enviar los pronunciamientos a pesar de no haber sido consultados oficialmente. Sobre la participación en audiencias las posibilidades aumentan, ya que los diputados pueden invitar a audiencia a quienes consideren prudente escuchar, o los participantes pueden solicitar audiencia a pesar de no haber sido invitados o pueden ir invitados por otros.

Considerando esta tipología de participación, es importante señalar que el Parlamento genera una interacción trazada por la desigualdad $y$ por ende, una disputa constante por ejercer el derecho a la expresión, en donde no todos los participantes tienen el mismo acceso al escenario legislativo. Esto también implica que la diferenciación existente entre los participantes se traduce en la asignación desigual de los momentos en los que pueden hacer uso de la palabra. Así, "no todos los turnos de habla tienen un valor semejante dentro de la situación de sesión parlamentaria; tampoco los hablantes, $y$ no cualquier diputado es encargado de presentar las iniciativas legislativas importantes" (Carbó, 1984a: 91).

Es por ello que existirían sujetos "que por una asignación de autoridad que se efectúa con criterios administrativos $y / 0$ políticos, pero no lingüísticos, desempeñan los cargos de Presidente y Secretario de sesiones y que son los que tienen el derecho y la obligación de usar un determinado tipo de turno de habla" (Carbó, 1984b: 112). Los hablantes de segundo orden serían "todos los demás participantes en la situación [... que...] son, en principio, semejantes $y$ tienen iguales oportunidades al uso del tipo de habla que les corresponde" (Carbó, 1984b: 112), pero que son diferentes y por tanto participan de manera desigual. 


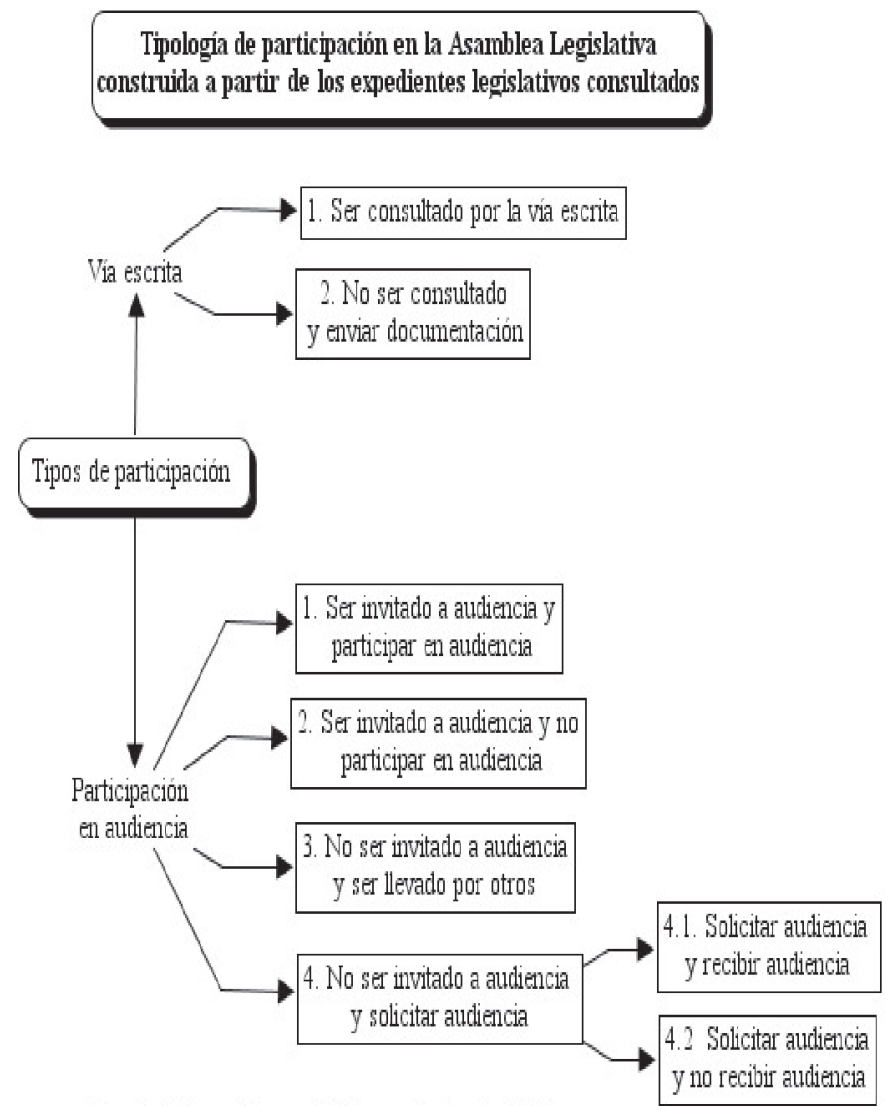

Fuente: Elaboración a partir de expedientes legislativos.

Esta distinción es relevante debido a que no todos los diputados se encuentran revestidos con la misma cuota de poder y no todos tienen la misma ingerencia en la toma de decisiones. Por el contrario, en las discusiones legislativas se puede identificar a diputados que asumen las riendas de las discusiones, mientras que otros carecen de voz, a pesar de ejercer el voto en el momento de decidir. De la misma manera, no todos los involucrados en la discusión tienen las mismas facilidades para ser escuchados. Por ello, participar en la Asamblea Legislativa, asumir determinadas funciones al interior de las comisiones y el ser portador de la palabra en una discusión, ilustra cómo se construye una participación desigualdad en el espacio legislativo.

\section{PARTICIPACIÓN LEGISLATIVA: LA DIFERENCIA SE CONVIERTE EN DESIGUALDAD}

El proyecto de ley de Protección a las Obtenciones Vegetales ingresó a la Comisión de Propiedad Intelectual el 28 de junio de 1999. Cuando esta comisión expiró en el año 2002, el proyecto fue trasladado a la Comisión de Asuntos Agropecuarios en donde se mantuvo hasta finales del 2005. Por su parte, el Convenio UPOV, que también había ingresado al parlamento en 1999, fue discutido en el 2002 en la Comisión de Relaciones Internacionales, en donde obtuvo su dictamen afirmativo de mayoría el 2 de julio del mismo año. En esta comisión se mantuvo hasta que fue archivado en el 2003. 
A diferencia de las iniciativas gubernamentales, el proyecto de ley propuesto por la Red de Coordinación en Biodiversidad en noviembre del 2003, no se había empezado a discutir a finales del 2005. Este tratamiento de la propuesta parlamentaria de las organizaciones sociales refleja el trato que también recibieron sus representantes en las comisiones legislativas, siendo este un primer nivel de la desigualdad analizada en la participación para aprobar proyectos de ley.

Analizando la participación al interior de las comisiones es central destacar el papel de tres diputados, lo que permite ilustrar prácticas y escenarios de construcción de consensos legislativos diversos. Belisario Solano del PUSC (19982002) fue una figura protagónica de la Comisión de Propiedad Intelectual durante 1999. Ante el rezago legislativo de los dos proyectos basados en la ley UPOV frente al "Combo de la Propiedad Intelectual", Solano prometió una amplia discusión legislativa sobre el tema a principios del 2000. Sin embargo, al no lograr consensos la discusión devino en inacción parlamentaria, por lo que el trámite legislativo se detuvo reactivándose hasta el 2002 con la Administración Pacheco de la Espriella. La ausencia de trámites parlamentarios durante el 2000 y el 2001 se debió al reconocimiento de Solano de la conflictividad existente en el tema, así como de la oposición de las organizaciones sociales. De la misma manera, las movilizaciones de marzo y abril del 2000 por la aprobación del Combo ICE incidieron en la tramitación de los proyectos UPOV, los que fueron considerados proyectos altamente conflictivos.

A partir del 2002, la nueva composición de la Asamblea Legislativa incidió en el tipo de discusiones y sujetos consultados. En este nuevo contexto se contrapusieron dos escenarios de participación y construcción de consensos políticos. Un primer escenario es el que propició el diputado Federico Malavassi del Movimiento Libertario en la Comisión de Relaciones Internacionales, comisión que discutía el Convenio UPOV. Malavassi potenció una participación acotada de los sujetos más calificados para referirse al tema, lo que excluiría a los grupos indígenas y a las organizaciones sociales, quienes desde su perspectiva tenían poco que aportar a una discusión sobre propiedad intelectual.

En este sentido, las críticas del Plenario en las figuras de José Miguel Corrales del PLN y
Gerardo Vargas del PAC se dirigieron a la rápida tramitación del proyecto, el tipo de sujetos consultados y las eventuales consecuencias de la aprobación del Convenio.

Enfrentando estas críticas Malavassi propuso la consulta a una serie de organizaciones para que estas enviaran sus criterios únicamente por la vía escrita. Las condiciones de la consulta fueron:

Por el máximo plazo que es de 8 días, para que envíen sus manifestaciones o expresiones a esta Comisión y con eso satisfacemos todas las solicitudes. A las comunidades indígenas, identificadas e inscritas, aunque yo no veo la pertinencia, pero también que se les haga consulta. Si ellos no la tramitan no es nuestro problema (Asamblea Legislativa, Expediente nro. 13756: 372).

Estas consultas tenían como finalidad evitar la impugnación ante las eventuales decisiones. Valga señalar que esta consulta fue dirigida a 17 organizaciones - la mayoría de carácter indígena-, de las cuáles sólo 3 respondieron a la consulta.

En contraposición, un segundo escenario de participación fue el que propició el PAC con la figura de Gerardo Vargas. Este diputado utilizó todos los recursos disponibles para convertir a la Asamblea Legislativa en un espacio de participación inclusivo, en donde fueran escuchadas las posiciones de los representantes de organizaciones sociales, indígenas y campesinas. Así, la ausencia de consultas a las organizaciones campesinas fue definido como:

Un irrespeto para los costarricenses, señor Presidente, que un proyecto de esta naturaleza sin haber sido consultado a organizaciones agrícolas, me refiero a la Corporación Hortícola Nacional, me refiero a UPANACIONAL (Asamblea Legislativa, Expediente nro. 13756: 358; cursivas en el original).

Consecuente con esta posición, Gerardo Vargas solicitó que el Convenio UPOV fuera reenviado a la comisión dictaminadora gracias 
al artículo 154 del Reglamento de la Asamblea Legislativa, para que se consultara a las organizaciones sociales. Posteriormente, este mismo diputado fue quien acogió en su despacho el proyecto de ley de Protección de los Derechos de los Fitomejoradores, iniciativa popular de la Red de Coordinación en Biodiversidad.

Este es el contexto en el que los sujetos participan para incidir políticamente en la tramitación de los proyectos, desplegando una serie de estrategias de apropiación del escenario legislativo, cuyas características expongo seguidamente.

\section{LAS ESTRATEGIAS DISCURSIVAS DE APROPIACIÓN DEL ÁMBITO LEGISLATIVO}

Las estrategias discursivas de apropiación del ámbito legislativo son mecanismos de presentación de los sujetos, estrategias utilizadas para asegurarse de ser escuchados por los diputados. La finalidad de estos mecanismos es dar validez a la participación y permiten evidenciar cómo se conciben a sí mismos los sujetos involucrados en la discusión, cómo conciben las temáticas tratadas en los proyectos de ley y finalmente, cómo conciben a quienes manifiestan posiciones contrarias. La argumentación fue utilizada como un instrumento que posibilita la inserción en el espacio legislativo, en donde se trata de convencer al otro, de que quién habla tiene autoridad para expresarse sobre el tema.

En las estrategias descritas a continuación destaco la participación de una serie de sujetos que se involucraron en las discusiones legislativas. Estos fueron los diputados de las comisiones legislativas, los representantes de la Oficina Nacional de Semillas, entidad estatal que presta servicios de control de calidad, certificación de semillas y registro de variedades importadas y exportadas por el país, el Grupo Técnico-Científico, grupo informal de investigadores de la Universidad de Costa Rica $^{5}$ y la Red de Coordinación en Biodiversidad, alianza de organizaciones compuesta por la Mesa Nacional Campesina, la Mesa Nacional Indí-

$\mathrm{Su}$ informalidad reside en que sus miembros no fueron representantes oficiales de una posición universitaria, como si lo sería el Consejo Universitario de la Universidad de Costa Rica. gena, la Federación de Conservación del Ambiente (FECON), el Programa CAMBIOS de la Universidad Nacional (UNA) y el Consejo de Defensa de la Institucionalidad (CDI).

Una primera estrategia utilizada fue la descalificación que los diputados hicieron de sí mismos. La dinámica suscitada en la Comisión de Propiedad Intelectual potenció el uso de esta estrategia. Al posicionarse como representantes del Poder Legislativo los diputados reconocieron el carácter técnico de los proyectos analizados lo que definió no solo el papel jugado por los diputados, sino el papel que debían jugar los consultados en el tema.

Las intervenciones del diputado Belisario Solano en 1999 lo ilustran:

... este campo es estrictamente especializado, donde el político lo único que tiene que hacer es tener la habilidad y la humildad para poder aceptar la recomendación que los técnicos y los expertos le pueden hacer. Yo le pido a Dios que me dé eso, que me dé esa humildad y esa habilidad para tratar de encausar la buena fe y el trabajo de ustedes hacia el futuro (Asamblea Legislativa, Expediente nro. 13640: 467).

Desde la perspectiva de Solano, un tema técnico y complejo requiere de los expertos que ayuden a entenderlo. Así, la función del diputado es tomar la mejor decisión de acuerdo con los consejos expresados por los expertos, ya que las decisiones políticas son sabias en la medida en que incorporan los criterios expuestos por los conocedores del tema. Sin embargo, no se apeló únicamente al criterio experto para llegar a una decisión política acertada. Lo divino en la toma de decisiones permitió al diputado reconocer que sobre temas muy especializados, no es él quien más conoce. De esta forma, la función de legislar aparece como un esfuerzo de generar consensos sólidamente construidos, fundamentados en las recomendaciones de los expertos y con la asistencia de Dios.

Es por ello que el criterio de los "expertos" fue una segunda estrategia para posicionarse en el ámbito legislativo. Esta estrategia se evidenció no solo en la necesidad expresada por los diputados 
de consultar a los conocedores del tema, sino también en el reconocimiento propio por parte de los expertos, quienes se ampararon en la necesidad de ser escuchados como tales.

De acuerdo con el Dr. William González ${ }^{6}$ "el tema de las obtenciones vegetales es un tema muy técnico, es un tema que compete básicamente a dos campos: por un lado al campo de la mejora genética $y$ biotecnología $y$, por otro lado, a la parte del derecho de propiedad intelectual" (Asamblea Legislativa, Expediente nro. 13640: 802). Una vez delimitadas las áreas de dominio de los expertos, estos se posicionaron como portadores del conocimiento válido a considerarse en la toma de decisiones, por lo que no todos los consultados fueron considerados bajo esa condición.

Los miembros del Grupo Técnico-Científico se denominaron:

Técnicos-científicos porque no existe o no hay un grupo debidamente establecido, sino que es un grupo de nueve profesionales científicos y otras personas del sector privado quienes nos hemos venido reuniendo porque estamos interesados en los aspectos de esta ley (Asamblea Legislativa, Expediente nro. 13640: 456).

De esta forma, la contraparte de esta estrategia fue la identificación de los desconocedores del tema, en donde alejados de la condición de especialistas fueron registrados los representantes de la Mesa Nacional Campesina.

Así, el diputado socialcristiano Mario Calderón definió al Dr. González como un "especialista en semillas, pero de la Mesa Campesina, quién es, qué conocimiento trae para hablar del tema" (Asamblea Legislativa, Expediente nro. 13640: 794). Es por estas razones que Eva Cara$\mathrm{zo}^{7}$ no cumplía con los requisitos para participar en las sesiones legislativas. Citando nuevamente

6 Fitomejorador de la Estación Experimental Fabio Baudrit de la UCR e integrante del Grupo TécnicoCientífico. Este grupo participó en las discusiones legislativas de 1999, en la Comisión de Propiedad Intelectual.

Eva Carazo representó a la Mesa Nacional Campesina en las discusiones legislativas. a Calderón: "Si alguien nos viene a hablar de un expediente, tiene que ser una persona que tenga conocimiento de aquello, esta muchacha Eva, lo que anda haciendo aquí es andar en todas las comisiones" (Asamblea Legislativa, Expediente nro. 13640: 794-795). Es así como en la configuración del espacio de debate de un proyecto de ley, los diputados reconocieron su ignorancia en el tema, lo que reforzó la necesidad de los expertos, quienes se encontraron en la mejor disposición de asumir el papel asignado por los diputados.

Sin embargo, ante el cuestionamiento de a quiénes representaron los expertos, surgió la estrategia de la doble adscripción a las instituciones públicas e intereses privados. Los funcionarios de las entidades universitarias se presentaron ante el auditorio legislativo, entre la ambigüedad de representar a las instituciones universitarias públicas y su posición personal.

Un ejemplo de esta estrategia fue la presentación hecha por el Dr. González:

Soy Fitomejorador, trabajo con la Universidad de Costa Rica, en la Estación Experimental Fabio Baudrit M., sin embargo en esta ocasión vengo en carácter personal, representando a un grupo de profesionales que nos hemos venido reuniendo con el fin de analizar algunos de los proyectos de ley (Asamblea Legislativa, Expediente nro. 13640: 456).

Estos sujetos se presentaron en nombre de las entidades universitarias en las que se desempeñan como investigadores, pero seguidamente aclararon que sus posiciones eran personales, reconociendo que no representaban la posición oficial de la UCR. De esa forma, discursivamente se conservó una ambigüedad en la presentación, que les permitió el ingreso a la Asamblea Legislativa.

No obstante, este ingreso no se dio sin conflictos. Los miembros del Grupo TécnicoCientífico fueron cuestionados en cuanto a la dificultad de identificar en nombre de quién hablaban sus integrantes. Ante este interrogante se recibieron dos tipos de respuestas. En la primera se aceptó que la posición presentada ante la comisión legislativa refería a criterios personales y por lo tanto privados, debido a que no existía respaldo institucional para los criterios expresados 
en la comisión legislativa. No obstante, una segunda respuesta reformuló argumentativamente el concepto de representación. De acuerdo con el Dr. González, si bien quienes hablan en nombre de las organizaciones son sus representantes, es necesario cuestionarse la importancia de esa representación en términos de su papel en la producción nacional del mejoramiento de variedades. Por ello se debe considerar no solo en nombre de cuántos se habla, sino en nombre de cuánto se produce (Asamblea Legislativa, Expediente nro. 13640: 490). Con este nuevo significado atribuido al concepto representación, las organizaciones sociales fueron descalificadas para hablar de semillas mejoradas y propiedad intelectual.

Ante el cuestionamiento de la representación, la recurrencia de los campesinos fue otra de las estrategias utilizadas tanto por los diputados, los universitarios como por los representantes de las organizaciones. El uso de esta estrategia se acentuó principalmente a partir del 2002, en donde el campesino apareció como el sujeto de la reflexión sobre propiedad intelectual. El "campesino" entendido como categoría cultural (Edelman, 2005: 339) permitió identificar la importancia política que este adquirió como referente abstracto del diálogo legislativo, en donde tanto científicos como diputados crearon un discurso en función del campesino.

Por parte de los diputados, Gerardo Vargas del PAC fue quien se asumió como el representante de las organizaciones sociales, definiéndose también como un agricultor. Dentro del ámbito legislativo, este diputado fue identificado como una voz representativa del sector agropecuario, siendo reconocido no solo como defensor de los agricultores, sino también como agricultor. Así, su asesor legislativo lo definió como "un pequeño agricultor de Cartago, pequeño agricultor que siembra hortalizas y que conoce bien la práctica de los agricultores en el manejo de las semillas" (Entrevista a José María Villalta, 28 de octubre del 2005). En el caso del discurso técnico-científico, el campesino se convirtió en el beneficiario de la aprobación de los proyectos. De acuerdo con la Dra. Ana Sittenfeld ${ }^{8}$, la aprobación de los proyec-

La Dra. Ana Sittenfeld del Centro de Biología Celular y Molecular de la UCR formó parte del Grupo Técnico-Científico. tos de ley tendrían como beneficiarios principalmente a los agricultores, ya que "la problemática para mí es solucionarle al campesino su problema, que es el productor, que es quien produce aquí en Costa Rica" (Entrevista a Ana Sittenfeld, 7 del noviembre de 2005). Sin embargo, Eva Carazo señaló como la representación de los campesinos es también uno de los principales recursos de las organizaciones sociales, recurso discursivo que no deja de ser una falacia argumentativa, ya que "en la práctica no era que 15000 familias de la Mesa Nacional Campesina, sintieran preocupación por el Convenio UPOV" (Entrevista a Eva Carazo, 25 de octubre del 2005).

Ahora bien, los representantes de las organizaciones, sin contar con el prestigio otorgado por la adscripción universitaria, ni la experiencia en el fitomejoramiento recurrieron a la estrategia que he denominado la sobredimensión de la representación. Estos se presentaron frente al auditorio legislativo hablando en nombre de la propia organización y en nombre de otras organizaciones que no estaban presentes en la Asamblea Legislativa. Esto se evidenció en la presentación en audiencia del Lic. Isaac Rojas:

Muchas gracias señor Presidente, soy abogado, pertenezco a la organización COECoceIBA-Amigos de la Tierra, Costa Rica. Igualmente vengo en representación tanto del Programa Cambios-Universidad Nacional y la Mesa Campesina que es un grupo y junto con don Rodrigo $\left[{ }^{9}\right]$, nos hemos dado a la tarea de generar información y reflexión sobre este tema que tanto nos preocupa (Asamblea Legislativa, Expediente nro. 13640: 352).

Directamente relacionada con esta estrategia, los representantes de las organizaciones se presentaron acompañados de otras personas que no fueron invitados a participar a las sesiones legislativas. Al llegar a la audiencia, aquellos invitados formalmente solicitaron a los diputados escuchar las posiciones de quienes no habían sido invitados, pero cuyo mensaje era de vital importancia para el desarrollo de la discusión.

Refiere a Rodrigo Carazo, expresidente de la República y presidente del Consejo de Defensa de la Institucionalidad (CDI). 
La utilización de ambas estrategias reflejó el establecimiento y permanencia de la Red de Coordinación en Biodiversidad, alianza surgida como una forma de contrarrestar las dificultades para participar en la Asamblea Legislativa.

\section{PARTICIPACIÓN DESIGUAL Y COMUNIDADES EPISTÉMICAS}

Quienes más facilidades tuvieron para ser escuchados, ya fuera por la vía escrita o la audiencia, fueron los representantes de instituciones públicas como la Oficina Nacional de Semillas y los funcionarios de universidades estatales como el Grupo Técnico-Científico. A pesar de que en las audiencias participaron representantes de instituciones estatales, entidades universitarias y organizaciones sociales, fueron estas últimas las que más dificultades tuvieron para recibir audiencia.

Así por ejemplo, en la discusión del proyecto de ley de Protección a las Obtenciones Vegetales, la Dra. Rodríguez ${ }^{10}$, los representantes de la Red de Coordinación en Biodiversidad y el Sr. Rodríguez, presidente de la Comisión Nacional para la Gestión de la Biodiversidad (CONAGEBIO) solicitaron audiencias tras no haber sido invitados. Sin embargo, ninguno recibió respuestas positivas a sus demandas.

Ahora bien, es importante señalar que recibir audiencia no fue garantía de recibir atención por parte de los diputados. El 5 de noviembre de 2002 se dio audiencia a los representantes del Consejo de Defensa de la Institucionalidad (CDI), la Mesa Nacional Campesina y la FECON. Evaluando la atención recibida, el Lic. Rodrigo Carazo criticó la insuficiencia del tiempo asignado para exponer sus consideraciones, así como la ausencia de diálogo entre los diputados y los representantes de las organizaciones. En sus palabras:

la 'precisa' de la Comisión impidió la posibilidad de que se me hicieran preguntas. Sentí que la Comisión quería ser atenta conmigo, tenerme allí lo menos posible y librarse de mi aporte contra UPOV.

10 La Dra. Silvia Rodríguez es socióloga del Programa CAMBIOS de la Universidad Nacional y participa en la Red de Coordinación en Biodiversidad.
Como costarricense, [...] sentí mucho la forma de recibirme: ni una sola pregunta, ni una sola oposición a mis palabras (Asamblea Legislativa, Expediente nro. 13756: 698).

De esta manera, el conocimiento portado por los sujetos y la oficialidad fueron los principales recursos a partir de los cuales se articuló la participación legislativa. La posesión de ambos recursos potenció el surgimiento de dos legitimidades distintas desde las que se posicionaron los sujetos en el escenario legislativo: una legitimidad institucionalizada con mayor probabilidad de acceso e incidencia política y la legitimidad de las organizaciones sociales, desprovista del prestigio que les impidió ser reconocidos como interlocutores válidos. Es la posesión o carencia de estos recursos lo que permite entender el tránsito desde la diferenciación hacia la desigualdad social, en donde los portadores de conocimientos "no científicos" y por lo tanto "diferentes", tuvieron menos posibilidades que otros de ser escuchados para incidir en la toma de decisiones.

El amplio debate surgido en las ciencias sociales sobre la relación entre el conocimiento especializado y la política ${ }^{11}$, invita a repensar la relación entre los tomadores de decisiones y la incidencia política de quienes portan conocimientos especializados ${ }^{12}$. Quizá uno de los

11 La obra clásica de la sociología en este tema es "El político y el científico" de Max Weber.

12 Algunas herramientas analíticas de interés son (1) la tecnocracia entendida como " "gobierno mediante la técnica»" (Meynaud, 1968: 37) (2) Los grupos de expertos o tanques de pensamientos, grupos de conocimientos especializados, vinculados a universidades, centros de estudios y unidades de investigación, que buscan la solución de problemas de política pública (Smith, 1994: 31). Y (3) las comunidades epistémicas. Haas desarrolla este concepto para explicar el surgimiento y funcionamiento del Plan de Acción Mediterráneo para el control de la contaminación. En este caso se constituyó una comunidad epistémica-ecologista, compuesta de profesionales como biólogos marinos, geógrafos, meteorólogos, químicos, así como ecologistas, que confiados en el método científico, identificaron las medidas necesarias para atender al problema de la contaminación del Mediterráneo (Haas, 1989: 384). 
acuerdos básicos en la producción sobre el tema, es que la mayor complejidad de los problemas políticos ha requerido de criterios cada vez más especializados para la resolución y toma de decisiones.

En este sentido, las dinámicas legislativas analizadas pueden entenderse como resultado de relación entre integrantes de comunidades epistémicas que se posicionaron en el escenario legislativo como portadores de visiones de mundo aglutinadas por conocimientos compartidos, a partir de los cuales validaron sus posiciones frente a los proyectos de ley. Compartiendo una serie de reglas, valores $y$ creencias provenientes de distintas disciplinas apostaron por soluciones diversas a la ausencia de regulación de la propiedad intelectual en Costa Rica (Haas, 1989: 380). Así, los miembros del Grupo Técnico-Científico y los representantes de la Oficina Nacional de Semillas se constituyeron como una comunidad epistémica portadora de conocimientos de la biotecnología, la agronomía o el derecho, cuyos integrantes poseen la experiencia y el prestigio asociado por desempeñarse en actividades de investigación en la Universidad de Costa Rica —en el primer caso- $y$ por contar con la oficialidad gubernamental —en el segundo caso- - Una segunda comunidad epistémica fue la compuesta por los integrantes de la Red de Coordinación en Biodiversidad, representantes del conocimiento de las ciencias sociales $y$ del derecho, pero también de las organizaciones sociales, quienes fueron percibidos por el resto de participantes como los desconocedores del tema.

Así, la construcción de derechos de propiedad intelectual se dirimió a través de la competencia de conocimientos y saberes especializados de estas dos comunidades epistémicas identificadas. Las discusiones sobre propiedad intelectual propiciaron el surgimiento de discursos de expertos - entendidos como los discursos de la biotecnología, la agronomía y el derecho-, constituidos como tales, no solo por la necesidad que los diputados tuvieron de ellos, sino también porque sus emisores asumieron el papel de especialistas en el tema. Los discursos de expertos se configuraron como discursos de prestigio $y$ de experiencia, circunscritos al ámbito de instituciones como las universidades estatales o las entidades públicas.

\section{CONCLUSIONES: INCIDENCIA POLÍTICA} DE LAS COMUNIDADES EPISTÉMICAS

Más allá del ámbito de la propiedad intelectual, esta reflexión plantea el problema de cómo nuestra sociedad construye el diálogo político y cómo construye a los interlocutores válidos con quiénes es prudente dialogar. De la misma manera, el trabajo invita a pensar con qué mecanismos se silencia a quiénes no son considerados como pares en una discusión. Por este motivo, el tema de la incidencia política tiene que ver no solo con los intereses que defienden los miembros de una comunidad epistémica, sino también con los valores y significados que recaen en determinados conocimientos y sus portadores.

La participación en la construcción de derechos de propiedad intelectual estuvo mediada por la legalidad del Reglamento de la Asamblea Legislativa, legalidad que promovió las consultas a los representantes gubernamentales, dificultando la participación de las organizaciones sociales. Sin embargo, la oficialidad no fue el único lugar que facilitó el acceso a la Asamblea Legislativa. Los miembros de las comunidades epistémicas fueron escuchados de acuerdo con el prestigio de la institucionalidad que dijeron abierta o indirectamente representar $y$ al prestigio asociado a sus conocimientos. En este sentido, las dinámicas legislativas analizadas expresan un conflicto no solo epistemológico y disciplinario, sino también político, en donde las posiciones sustentadas en conocimientos de las organizaciones y las ciencias sociales, fueron constantemente interpeladas como no válidas.

Si bien las organizaciones sociales tuvieron más dificultades para expresar sus criterios en las instancias legislativas, a finales del 2005 los integrantes de la Red de Coordinación en Biodiversidad se definieron como los más influyentes en la toma de decisiones. De acuerdo con la Dra. Rodríguez, la Red de Coordinación en Biodiversidad, con el apoyo del Lic. Rodrigo Carazo y el diputado Gerardo Vargas, habrían sido los más influyentes al lograr detener el proceso de aprobación del Convenio UPOV y entrabar la aprobación del proyecto de ley de Protección a las Obtenciones Vegetales. Sin embargo, hasta ese momento el proyecto de ley de Protección de los Derechos de los Fitomejoradores, no había 
sido discutida en la Comisión de Asuntos Agropecuarios.

En este sentido, dicha ingerencia debe contextualizarse y por ende, no se debe olvidar que la tramitación de los proyectos de ley analizados se vio entrecruzada por las negociaciones $y$ las presiones que ejercieron diversos sectores para que la Administración Pacheco de Espriella enviara el Tratado de Libre Comercio entre Centroamérica y Estados Unidos (TLC) a la Asamblea Legislativa. De esta manera, será en el contexto de la tramitación del TLC cuando se pueda determinar efectivamente qué comunidades epistémicas fueron más influyentes en la construcción de derechos de propiedad intelectual sobre variedades mejoradas.

\section{BIBLIOGRAFÍA}

Asamblea Legislativa. "Expediente nro. 13156 Tratado de la OMPI sobre interpretación y Ejecución de Fonogramas". (San José: Asamblea Legislativa). 1998.

Asamblea Legislativa. "Expediente nro. 13157 Tratado de la OPMI sobre Derechos de Autor". (San José: Asamblea Legislativa). 1998.

Asamblea Legislativa. "Expediente nro. 13640 Proyecto de Ley de Protección a Obtenciones Vegetales". (San José: Asamblea Legislativa). 1999.

Asamblea Legislativa. "Expediente nro. 13641 Proyecto de Ley de Marcas y Signos distintivos". (San José: Asamblea Legislativa). 1999.

Asamblea Legislativa. "Expediente nro. 13642 Proyecto de Ley de Procedimientos de Observancia de los Derechos de Propiedad Intelectual". (San José: Asamblea Legislativa). 1999.

Asamblea Legislativa. Expediente nro. 13643 Proyecto de Ley Reformas a la ley de
Derechos de Autor y Derechos Conexos nro. 6683 y sus reformas". (San José: Asamblea Legislativa). 1999.

Asamblea Legislativa. "Expediente nro. 13643 Proyecto de Ley Reformas a la ley Patentes de Invención, Dibujos y Modelos Industriales y Modelos de Utilidad nro. 6867 y sus reformas". (San José: Asamblea Legislativa). 1999.

Asamblea Legislativa. "Expediente nro. 13711 Proyecto de Ley de Protección a los sistemas de Trazado de Circuitos Integrados". (San José: Asamblea Legislativa). 1999.

Asamblea Legislativa. Expediente nro. 13756 Proyecto de Ley Convenio Internacional para la Protección de Obtenciones Vegetales”. (San José: Asamblea Legislativa). 1999.

Asamblea Legislativa. "Expediente nro. 15487 Proyecto de Ley de Protección de los Derechos de los Fitomejoradores". (San José: Asamblea Legislativa). 1999.

Asamblea Legislativa. "Reglamento de la Asamblea Legislativa". (San José: Asamblea Legislativa). 2001.

Carbó, Teresa. "Acerca de un discurso político en proceso de constitución. El departamento de Educación y Cultura para la Raza Indígena: 1921". Cuadernos de la Casa Chata "Discurso político: Lectura y Análisis”. México. Nro. 5. 1984a.

"Un análisis de Discurso Parlamentario. Debatiendo sobre los otomíes (1952)". Cuadernos de la Casa Chata "Discurso politico: Lectura y Análisis”. México, nro. 5. 1984b.

Edelman, Marc. Campesinos contra la globalización. Movimientos sociales rurales en Costa Rica. 1 edición, San 
José: Editorial de la Universidad de Costa Rica. 2005. 453 páginas.

Haas, Peter. "Do Regimes Matter? Epistemic Communities and Mediterranean Pollution". Internacional Organization. Cambridge, vol. 43, nro. 3.

Meynaud, Jean. Tecnocracia ¿Mito o realidad?. lera edición, Madrid: Editorial Tecnos, 393 páginas. 1968.
Smith, James. (1991). Intermediarios de ideas. Los "Grupos de Expertos (Think Tanks) y el surgimiento de la nueva élite política”. 2 impresión, Argentina: Grupo Editor Latinoamericano, 1994. 292 páginas.

Weber, Max. 1919. El político y el científico. 15 reimpresión, Madrid: Alianza Editorial, 1995. 233 páginas. 